\title{
Robust Adaptive Nonlinear Kalman Filter for Synchronous Machine Parameter Calibration
}

\begin{tabular}{|c|c|c|c|c|}
\hline $\begin{array}{c}\text { Junbo Zhao } \\
\text { MSU } \\
\text { unbo@ece.msstate.edu }\end{array}$ & $\begin{array}{c}\text { Shaobu Wang, Renke Huang } \\
\text { PNNL } \\
\text { u shaobu.wang@pnnl.gov }\end{array}$ & $\begin{array}{l}\text { Rui Fan } \\
\text { University of Denver } \\
\text { rui.fan@ du.edu }\end{array}$ & $\begin{array}{c}\text { Yijun Xu } \\
\text { Virginia Tech } \\
\text { yijunxu@vt.edu }\end{array}$ & $\begin{array}{c}\text { Zhenyu Huang } \\
\text { PNNL } \\
\text { zhenyu.huang@ } @ \text { pnnl.gov }\end{array}$ \\
\hline
\end{tabular}

\begin{abstract}
This paper proposes a robust and adaptive nonlinear Kalman filter for synchronous machine parameter calibration. The key idea is to develop the polynomial chaos-based analysis of variance (ANOVA) method for suspicious parameter detection. ANOVA allows us to derive a set of adaptive weights that can be used to address local parameter optimality issue when performing joint state and parameter estimation. It is shown that if erroneous parameters have strong correlations, the widely used methods that augment state and parameter for joint estimation will lead to large biases. By contrast, thanks to the derived adaptive weights for the suspicious parameters, the proposed method can effectively deal with the parameter dependence, yielding much better calibration results. In addition, the robustness of the proposed method enables us to filter non-Gaussian noise. Simulations carried out on the IEEE 39-bus system validate the effectiveness and robustness of the proposed approach.
\end{abstract}

\section{Introduction}

In 1996, a major blackout occurred in the US Western Electricity Coordinating Council (WECC) system. According to the detailed post event analysis, the critical reason is that the simulated results from the models do not match the actual responses, which leads to wrong decision makings. On the other hand, the North American Electric Reliability Corporation (NERC) requires generator owners to validate and calibrate their models and associated controllers every five years $[1,2]$. This becomes particular important with the increasing penetration of renewable energy integration. Indeed, the synchronous machines are typically used as picking units to mitigate renewable generation stochasticity and uncertainties, and if their models are not reliable, the controls can be misleading that may cause cascading failures.

There are two main ways of calibrating generator parameters [3]. The first one is to shut down the generator and perform detailed field tests [4]. Although this would yield very accurate results, it is time consuming and costly. By contrast, with the wide-area deployment of phasor measurement units (PMUs), measurement-based methods are paid increasingly attention. The key idea is to leverage the generator point of coupling voltage phasors as generator model inputs and compare the model outputs with the measured values [5]. If there is a large discrepancy, the model is declared as invalid and parameter calibration is required. To find suspicious parameters, trajectory sensitivity analysis has been widely used $[6,7]$. After that, the suspicious parameters are augmented together with the states for joint estimation. To this end, many different methods have been developed [8]. In [9], the least square method is used to estimate inertia constant and primary frequency control droop of a synchronous generator. While in [10], the extended Kalman filter (EKF) is developed for mechanical power, inertia constant, damping factor and transient reactance estimation. To deal with the weakness of EKF in handling the system strong nonlinearity, unscented Kalman filter (UKF) [11,12] and ensemble Kalman filter (EnKF) [13] have been proposed for joint state and parameter estimation. Optimization methods based on heuristic approach, such as particle swarm optimization [14], and Bayesian inference [15] have also been developed. However, these methods will achieve poor performance when the suspicious parameters have strong dependence and in the presence of non-Gaussian measurement noise.

To address the aforementioned challenges, this paper proposes a robust and adaptive nonlinear UKF for synchronous machine parameter calibration. It has the following contributions:

- The generalized polynomial chaos-based analysis of variance (ANOVA) method is developed to detect suspicious parameters and investigate the parameter dependence. Analysis results from the ANOVA are further used to derive a set of 
adaptive weights that can be used to address local parameter optimality issue when performing joint state and parameter estimation;

- The adaptive weights have been integrated with the robust UKF to achieve good robustness against non-Gaussian noise. Comparison results with UKF and EnKF are presented to demonstrate the effectiveness and robustness of the proposed method.

The remainder of the paper is organized as follows: Section II presents the problem formulation while Section III describes the proposed robust and adaptive parameter estimator. Section IV analyzes the simulation results, and finally Section V concludes the paper.

\section{Problem Formulation}

The synchronous machine dynamics can be described by a set of differential and algebraic equations. Without loss of generality, the two-axis generator model with IEEE-DC1A exciter and TGOV1 turbine governor is used as an example in this paper. Formally, we have [16]:

Differential equations of generator:

$$
\begin{gathered}
\frac{d \delta}{d t}=\omega-\omega_{s}, \\
\frac{2 H}{\omega_{s}} \frac{d \omega}{d t}=T_{M}-P_{e}-D\left(\omega-\omega_{s}\right), \\
T_{d o}^{\prime} \frac{d E_{q}^{\prime}}{d t}=-E_{q}^{\prime}-\left(X_{d}-X_{d}^{\prime}\right) I_{d}+E_{f d}, \\
T_{q o}^{\prime} \frac{d E_{d}^{\prime}}{d t}=-E_{d}^{\prime}-\left(X_{q}-X_{q}^{\prime}\right) I_{q},
\end{gathered}
$$

Differential equations of IEEE-DCIA exciter:

$$
\begin{gathered}
T_{E} \frac{d E_{f d}}{d t}=-\left(K_{E}+S_{E}\left(E_{f d}\right)\right) E_{f d}+V_{R} \\
T_{\mathrm{F}} \frac{d V_{F}}{d t}=-V_{F}+\frac{K_{F}}{T_{E}} V_{R}-\frac{K_{F}}{T_{E}}\left(K_{E}+S_{E}\left(E_{f d}\right)\right) E_{f d} \\
T_{A} \frac{d V_{R}}{d t}=-V_{R}+K_{A}\left(V_{r e f}-V_{F}-V\right)
\end{gathered}
$$

Differential equations of TGOVI turbine-governor:

$$
\begin{gathered}
T_{C H} \frac{d T_{M}}{d t}=-T_{M}+P_{S V} \\
T_{S V} \frac{d P_{S V}}{d t}=-P_{S V}+P_{C}-\frac{1}{R_{D}}\left(\frac{\omega}{\omega_{s}}-1\right),
\end{gathered}
$$

Algebraic equations:

$$
\begin{gathered}
V_{d}=V \sin (\delta-\theta), V_{q}=V \cos (\delta-\theta), \\
I_{d}=\frac{E_{q}^{\prime}-V_{q}}{X_{d}^{\prime}}, I_{q}=\frac{V_{d}-E_{d}^{\prime}}{X_{q}^{\prime}}, \\
P_{e}=V_{d} I_{d}+V_{q} I_{q}, Q_{e}=-V_{d} I_{q}+V_{q} I_{d},
\end{gathered}
$$

where $\delta$ and $\omega$ are the rotor angle and speed, respectively; $\omega_{S}$ is the nominal synchronous speed; $T_{d o}^{\prime}, T_{q o}^{\prime}, T_{E}, T_{F}, T_{A}, T_{C H}$ and $T_{S V}$ are time constants, in seconds; $K_{E}, K_{F}$ and $K_{A}$ are controller gains; $V_{\text {ref }}$ and $P_{C}$ are known control inputs; $E_{q}^{\prime}$, $E_{d}^{\prime}, E_{f d}, V_{F}, V_{R}, T_{M}$ and $P_{S V}$ are the q-axis and d-axis transient voltages, field voltage, scaled output of the stabilizing transformer and scaled output of the amplifier, synchronous machine mechanical torque and steam valve position, respectively; $X_{d}, X_{d}^{\prime}, X_{q}$ and $X_{q}^{\prime}$ are generator parameters; $V$ and $\theta$ are the terminal bus voltage magnitude and phase angle, respectively; $P_{e}$ and $Q_{e}$ are the active and reactive electrical power outputs; $V_{d}$ and $V_{q}$ are the $\mathrm{d}$ and $\mathrm{q}$ axis voltage magnitudes, respectively; $I_{d}$ and $I_{q}$ are the $\mathrm{d}$ and $\mathrm{q}$ axis currents, respectively.

The above differential and algebraic equations can be discretized and the following discrete-time state space form is derived:

$$
\begin{gathered}
\boldsymbol{x}_{k}=\boldsymbol{f}\left(\boldsymbol{x}_{k-1}, \boldsymbol{p}_{k}, \boldsymbol{u}_{k}\right)+\boldsymbol{w}_{k}, \\
\boldsymbol{z}_{k}=\boldsymbol{h}\left(\boldsymbol{x}_{k}, \boldsymbol{p}_{k}\right)+\boldsymbol{v}_{k},
\end{gathered}
$$

where (13) and (14) correspond to equations (1)-(9) and (10)-(12), respectively; $\boldsymbol{x}_{k}$ is the state vector that includes state variables of the synchronous generator, the exciter and the governor; $\boldsymbol{z}_{k}$ is the measurement vector that contains generator terminal real and reactive power; $\boldsymbol{p}_{k}$ is the parameter vector that includes all the 22 parameters of generator and its associated exciter and governor; the noises $\boldsymbol{w}_{k}$ and $\boldsymbol{v}_{k}$ are assumed to be white and with covariance matrices $\boldsymbol{Q}_{k}$ and $\boldsymbol{R}_{k}$, respectively; $\boldsymbol{u}_{k}$ represents the input vector; $\boldsymbol{f}(\cdot)$ and $\boldsymbol{h}(\cdot)$ are the vector-valued functions. Since $V_{\text {ref }}$ and $P_{C}$ are locally controlled, they are typically assumed to be known parameters.

When there is an event occurring in the system, the generator terminal voltage phasor can be leveraged as $\boldsymbol{u}$ and substituted into the differential and algebraic equations. The resulted $P$ and $Q$ from the model are compared with the measured quantities to determine whether the model is adequate. If the difference is statistically significant, suspicious parameters need to be found out and calibrated. There are two challenges for this decentralized parameter calibration framework: 
1) the identification of suspicious parameters can be challenging if the erroneous parameters are strongly dependent with each other; 2) the PMU measurement errors are not necessarily Gaussian. This paper proposes a robust and adaptive UKF to address these challenges, which are shown in the next section.

\section{Proposed Robust and Adaptive Filter for Parameter Calibration}

In this section, a generalized regression form is first derived, which allows carrying out sensitivity analysis and suspicious parameter detection. Then, adaptive weights are derived for joint suspicious parameter calibration and state estimation.

\subsection{Derivation of Generalized Regression Form}

Following the UKF framework, if the initial state estimate $\widehat{\boldsymbol{x}}_{k-1 \mid k-1} \in \mathbb{R}^{n \times 1}$ and its covariance matrix $\Sigma_{k-1 \mid k-1}^{x x}$ at time step $k-1$ is given, $2 n$ weighted sigma points can be generated via

$$
\chi_{k-1 \mid k-1}^{j}=\widehat{\boldsymbol{x}}_{k-1 \mid k-1} \pm\left(\sqrt{n \boldsymbol{\Sigma}_{k-1 \mid k-1}^{x x}}\right)_{j}
$$

where $w_{j}=1 / 2 n, j=1, \ldots, 2 n$. Then $\chi_{k \mid k-1}^{(j)}$ is propagated through the nonlinear model (13) to obtain the transformed samples:

$$
\chi_{k \mid k-1}^{j}=\boldsymbol{f}\left(\chi_{k-1 \mid k-1}^{(j)}\right)
$$

Next, the predicted state $\widehat{\boldsymbol{x}}_{k \mid k-1}$ and its covariance matrix $\Sigma_{k \mid k-1}^{x x}$ are obtained through

$$
\begin{gathered}
\widehat{\boldsymbol{x}}_{k \mid k-1}=\sum_{j=1}^{2 n} w_{j} \boldsymbol{\chi}_{k \mid k-1}^{(j)}, \quad g\left(\xi_{1}, \ldots, \xi_{N}\right)=g_{0}+\sum_{1 \leq j_{1} \leq N} g_{j_{1}}\left(\xi_{j_{1}}\right) \\
\boldsymbol{\Sigma}_{k \mid k-1}^{x x}=\sum_{j=1}^{2 n} w_{j}\left(\boldsymbol{\chi}_{k \mid k-1}^{(j)}-\widehat{\boldsymbol{x}}_{k \mid k-1}\right)\left(\boldsymbol{\chi}_{k \mid k-1}^{(j)}-\widehat{\boldsymbol{x}}_{k \mid k-1}\right)^{T}+\boldsymbol{Q}_{k} \cdot+\sum_{1 \leq j_{1}<j_{2} \leq N} g_{j_{1}, j_{2}}\left(\xi_{j_{1}}, \xi_{j_{2}}\right)+\ldots+g_{1,2, \ldots, N} .
\end{gathered}
$$

By processing the predicted state vector and the measurement vector together, we get the following batch-mode regression form:

$$
\left[\begin{array}{c}
\boldsymbol{z}_{k} \\
\widehat{\boldsymbol{x}}_{k \mid k-1}
\end{array}\right]=\left[\begin{array}{c}
\boldsymbol{h}\left(\boldsymbol{x}_{k}, \boldsymbol{p}_{k}, \boldsymbol{u}_{k}\right) \\
\boldsymbol{x}_{k}
\end{array}\right]+\left[\begin{array}{c}
\boldsymbol{v}_{k} \\
\boldsymbol{\delta}_{k}
\end{array}\right]
$$

where $\boldsymbol{\delta}_{k}$ is the state prediction error that has zero mean and covariance matrix $\Sigma_{k \mid k-1}^{x x}$. (17) can be rewritten in a compact form as

$$
\boldsymbol{y}_{k}=\boldsymbol{g}\left(\boldsymbol{x}_{k}, \boldsymbol{p}_{k}, \boldsymbol{u}_{k}\right)+\boldsymbol{e}_{k} .
$$

This generalized regression form provides us a convenient way of conducting sensitivity analysis and state filtering.

\subsection{Sensitivity Analysis and Adaptive Weights Derivation}

It is worth pointing out that in (18), $\boldsymbol{x}_{k}, \boldsymbol{u}_{k}$ and the erroneous parameters of $\boldsymbol{p}_{k}$ are all random variables. For the generalized polynomial chaos (gPC) method, the stochastic outputs can be represented as a weighted sum of a given set of orthogonal polynomial chaos basis functions. The latter are derived from the probability distribution of the input random variables. For example, let $\boldsymbol{y}_{k}$ the output vector, $\boldsymbol{\xi}=\left[\xi_{1}, \xi_{2}, \ldots \xi_{N}\right]$ a vector of random variable, which in this paper represents $\boldsymbol{p}_{k}$ when $\boldsymbol{x}_{k}$ and $\boldsymbol{u}_{k}$ are replaced by the predicted state vector and generator terminal voltage phasor, $\phi_{i}\left(\xi_{1}, \xi_{2}, \ldots \xi_{N}\right)$ the polynomial chaos basis and $a_{i}$ the $i$ th polynomial chaos coefficient. Thus, we have the following expansion [17]:

$$
y=\sum_{i=0}^{\infty} a_{i} \phi_{i}(\boldsymbol{\xi})
$$

From the polynomial chaos coefficients, the mean, $\mu$, and the variance, $\sigma^{2}$, of the output $y$ can be easily calculated via

$$
\mu=a_{0}, \sigma^{2}=\sum_{i=1}^{\infty} a_{i}^{2} E\left[\phi_{i}^{2}\right]
$$

where $E[$.$] is the expectation operator.$

On the other hand, according to ANOVA, a nonlinear function $g$ can be expanded with $N$ random variable as follows [17]:

where $\xi_{i}, i=1, \ldots, N$ are random variables following a known probability; the functions $g_{j_{k}}\left(\xi_{j_{k}}\right), 1 \leq$ $j_{k} \leq N, g_{j_{k}, j_{l}}\left(\xi_{j_{k}}, \xi_{j_{l}}\right), 1 \leq j_{k} \leq j_{l} \leq N$, etc., are the so-called first-order, second-order,... ANOVA components, respectively. Define $T_{j_{1}, \ldots, j_{s}}$ as the fraction of the variance $\sigma^{2}(g)$ contributed by $g_{j_{1}, \ldots, j_{s}}$, we have

$$
T_{j_{1}, \ldots, j_{s}}=\frac{\sigma^{2}\left(g_{j_{1}, \ldots, j_{s}}\right)}{\sigma^{2}(g)} .
$$

where $T_{j_{1}}$ indicates that the variance is only contributed by $\xi_{j_{1}} ; T_{j_{1}, j_{2}}$ is the variance contributed by the 
dependence of $\left(\xi_{j_{1}}, \xi_{j_{2}}\right)$. Note that $T_{j_{1}, \ldots, j_{s}}$ satisfies the following constraint:

$$
\sum_{i=1}^{N} T_{j_{1}}+\sum_{1 \leq j_{1}<j_{2} \leq N} T_{j_{1}, j_{2}}+\ldots T_{j_{1}, \ldots, j_{N}}=1
$$

To find the relationship between gPC and ANOVA, (19) is expanded to the following functional form:

$$
\begin{aligned}
& y=a_{0}+\sum_{i=1}^{N} a_{i} \phi\left(\xi_{i}\right)+\sum_{i=1}^{N} \sum_{j=1}^{i} a_{i, j} \phi\left(\xi_{i}, \xi_{j}\right) \\
& +\sum_{i=1}^{N} \sum_{j=1}^{i} \sum_{r=1}^{j} a_{i, j, r} \phi\left(\xi_{i}, \xi_{j}, \xi_{r}\right)+\ldots
\end{aligned}
$$

In practice, a truncated version of $\mathrm{gPC}$ is used, i.e.,

$$
y=\sum_{i=0}^{N_{l}} a_{i} \phi_{i}(\boldsymbol{\xi})
$$

where $N_{l}=(N+l) ! /(N ! l !)-1$ and $N$ is the total number of the random variables; $l$ is the maximum order of the polynomial chaos basis functions and this value is typically set as 2 in the literature [18]. As a result, we can obtain

$$
\begin{aligned}
& y=a_{0}+\left(\sum_{i=1}^{N} a_{i} \phi\left(\xi_{i}\right)+\sum_{i=1}^{N} \sum_{j=i} a_{i, j} \phi\left(\xi_{i}, \xi_{j}\right)\right) \\
& +\sum_{i=1}^{N} \sum_{1 \leq j<i} a_{i, j} \phi\left(\xi_{i}, \xi_{j}\right) .
\end{aligned}
$$

By comparing the gPC expansion with that of the ANOVA, we find that the first two summations and the third summation represent respectively the first-order and the second-order ANOVA components. To this end, (22) can be calculated through

$$
\begin{gathered}
T_{j_{1}}=\frac{\sigma^{2}\left(g_{j_{1}}\right)}{\sigma^{2}(g)}=\frac{a_{i}^{2} E\left[\phi\left(\xi_{i}\right)^{2}\right]+a_{i, j}^{2} E\left[\phi\left(\xi_{i}, \xi_{j}\right)^{2}\right]_{i=j}}{\sigma^{2}}, \\
T_{j_{1}, j_{2}}=\frac{\sigma^{2}\left(g_{j_{1}, j_{2}}\right)}{\sigma^{2}(g)}=\frac{a_{i, j}^{2} E\left[\phi\left(\xi_{i}, \xi_{j}\right)^{2}\right]_{i \neq j}}{\sigma^{2}}
\end{gathered}
$$

By using the above two equations, the sensitivity of each parameter to the response $y$ can be analytically determined. In this paper, only those values that are larger than a certain threshold will be detected as suspicious parameters for further calibration. According to the suggestion in [18], the threshold for $T_{j 1}$ is usually set as $0.1 \%$. Note that $T_{j 1, j 2}$ is much smaller than $T_{j 1}$. In this paper, in order to reduce the number of calibrating less sensitive parameters, the $1 \%$ threshold is assumed.

During the estimation process using nonlinear Kalman filter, the value of $T_{j_{i}}$ will be taken as the weight of each parameter. This is because those parameters who have higher sensitivity to an event can be calibrated in a much easier manner and thus they should be assigned with a higher weight. The value of $T_{j_{i}}$ naturally reflects that. On the other hand, since $T_{j_{1}, j_{2}}$ reveals the level of dependence of two parameters, if this value is large, we propose to break the dependence to avoid the local optimality. This can be done as follows:

- Step 1: if there are several parameters that are identified to have strong dependence, they will be divided into two different groups;

- Step 2: one group of parameters will be used together with the model states for joint estimation while the other group remains unchanged;

- Step 3: once the estimation of the first group of parameters is done, they will be kept unchanged while augmenting the other group with model states for joint estimation;

- Step 4: both groups of parameters are augmented with model state for joint estimation, which will further improve the accuracy of the estimated parameters.

\subsection{Joint State and Parameter Estimation}

By applying the statistical linerization [19] to the nonlinear measurement function $\boldsymbol{h}(\cdot)$ around $\widehat{\boldsymbol{x}}_{k \mid k-1}$, we can get

$$
\boldsymbol{z}_{k}=\boldsymbol{H}_{k}\left(\boldsymbol{x}_{k}-\widehat{\boldsymbol{x}}_{k \mid k-1}\right)+\widehat{\boldsymbol{z}}_{k \mid k-1}+\boldsymbol{v}_{k}+\boldsymbol{\varsigma}_{k},
$$

where $\widehat{\boldsymbol{z}}_{k \mid k-1}=\sum_{j=1}^{2 n} w_{j} \boldsymbol{z}_{k \mid k-1}^{(j)}$ is the predicted measurement vector; $\boldsymbol{z}_{k \mid k-1}^{j}=\boldsymbol{h}\left(\boldsymbol{\chi}_{k \mid k-1}^{(j)}\right) ; \boldsymbol{H}_{k}=$ $\left(\boldsymbol{\Sigma}_{k \mid k-1}^{x z}\right)^{T}\left(\boldsymbol{\Sigma}_{k \mid k-1}^{x x}\right)^{-1}$ is the statistical linerization regression matrix, where

$\boldsymbol{\Sigma}_{k \mid k-1}^{x z}=\sum_{j=1}^{2 n} w_{j}\left(\boldsymbol{\chi}_{k \mid k-1}^{j}-\widehat{\boldsymbol{x}}_{k \mid k-1}\right)\left(\boldsymbol{z}_{k \mid k-1}^{j}-\widehat{\boldsymbol{z}}_{k \mid k-1}\right)^{T}$

and $\varsigma_{k}$ is the statistical linearization error who has zero mean and a covariance matrix that can be found in [19]. 


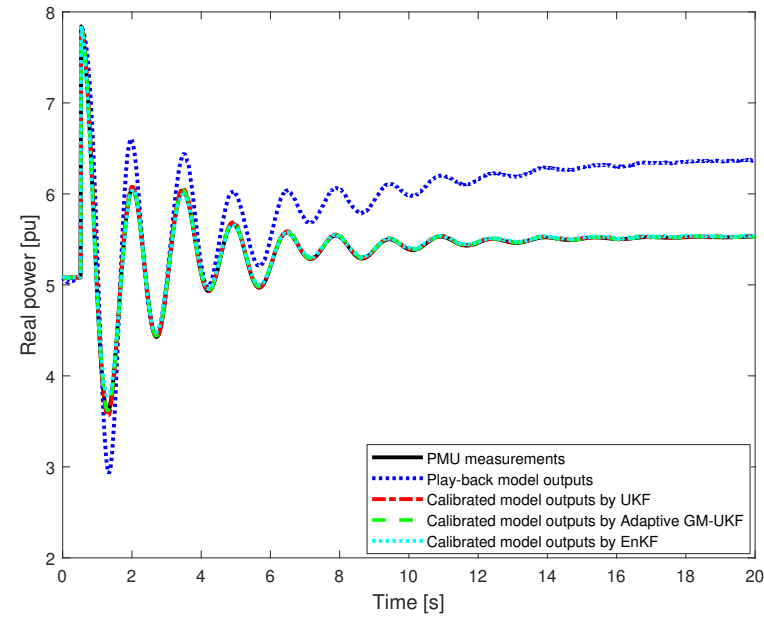

Figure 1. Real power response using calibrated parameters with adaptive weights.

Define a generalized state vector $\boldsymbol{x}_{k}^{g}=\left[\begin{array}{ll}\boldsymbol{x}_{k}^{T} & \boldsymbol{p}_{k}^{T}\end{array}\right]^{T}$ that includes the state vector and the suspicious parameters. Then, by processing the predicted state vector and the measurement vector together, we get

$$
\left[\begin{array}{c}
\boldsymbol{z}_{k}+\boldsymbol{H}_{k} \widehat{\boldsymbol{x}}_{k \mid k-1}-\widehat{\boldsymbol{z}}_{k \mid k-1} \\
\widehat{\boldsymbol{x}}_{k \mid k-1} \\
\boldsymbol{p}_{k \mid k-1}
\end{array}\right]=\left[\begin{array}{cc}
\boldsymbol{H}_{k} & \mathbf{0} \\
\boldsymbol{I} & \mathbf{0} \\
\mathbf{0} & \boldsymbol{I}
\end{array}\right] \boldsymbol{x}_{k}^{g}+\left[\begin{array}{c}
\boldsymbol{v}_{k}+\boldsymbol{\varsigma}_{k} \\
\boldsymbol{\delta}_{k} \\
\boldsymbol{\varepsilon}_{\boldsymbol{k}}
\end{array}\right]
$$

where $\boldsymbol{I}$ is an identity matrix with appropriate dimension. The above equation can be further rewritten in a compact form as

$$
\widetilde{\boldsymbol{z}}_{k}=\widetilde{\boldsymbol{H}}_{k} \boldsymbol{x}_{k}+\widetilde{\boldsymbol{e}}_{k},
$$

Following our previous work [19], the generalized maximum-likelihood estimator will be applied to (31) for joint state and parameter estimation.

Remark: It should be noted that since the accuracy of the initial generator parameter is unknown while the estimated generator states have much higher accuracy than the parameters, the variances of the parameters at the very beginning should be much larger than those associated with the states. In this paper, the variances of the suspicious parameters are set to be 100 times larger than the states.

\section{Numerical Results}

Case studies are carried out on the IEEE 39-bus system to evaluate the effectiveness and robustness of the proposed method. To simulate a system event, a three-phase short circuit occurs at $t=0.5 \mathrm{~s}$ for the line 33 and is cleared after 2 cycles by opening the

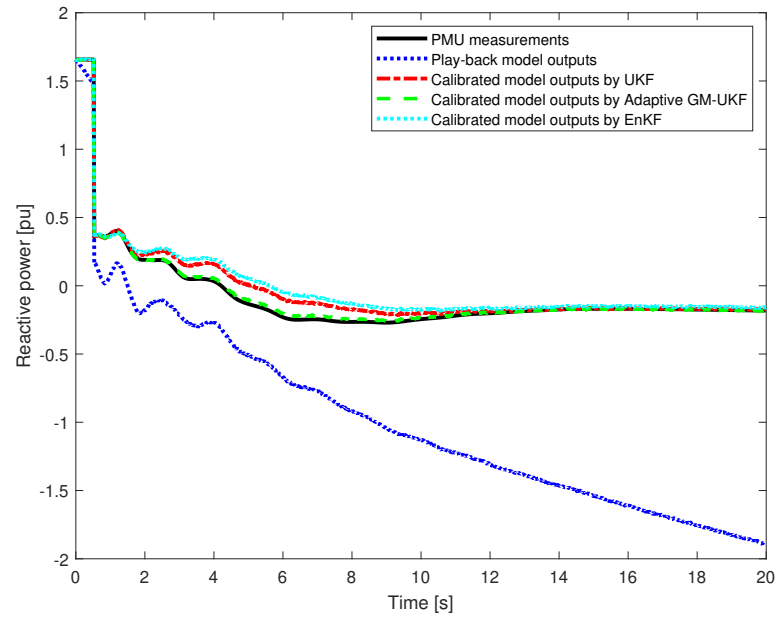

Figure 2. Reactive power response using calibrated parameters with adaptive weights.

transmission line 33-19. The time-domain simulation results are taken as the true values. The simulated voltage phasor and the calculated real and reactive power at each generator's terminal bus are treated as PMU measurements. All synchronous generators assumed for transient simulation are represented by the detailed two-axis generator model with IEEE-DC1A exciter and TGOV1 turbine-governor, whose parameter values are taken from [20]. All simulations are conducted 100 times and the average value is taken as the final estimate. Three methods are tested and compared, namely the traditional UKF and EnKF by augmenting the suspicious parameters with states for joint estimation, and the proposed robust and adaptive UKF, called Adaptive generalized maximum-likelihood UKF (GM-UKF). The initial states are calculated from the power flow results under the steady-state before the transient simulations and their associated covariance matrix is $10^{-6} \boldsymbol{I}$ with appropriate dimensions. $1 \%$ error for PMU measurements is assumed and the random variable can be generated via: $1 \% \times \mu_{m} / 3 \times \operatorname{rand}(m, 1)$ under the $99.7 \%$ confidence interval, where the mean $\mu_{m}$ is the true value of the original measurement; $\operatorname{rand}(m, 1)$ represents an $m \times 1$ dimension random variable that follows the standard normal distribution and $m$ is the number of measurements. The initial parameter values are chosen using the current dataset recorded in the planning department. It is worth pointing out that these parameters are physical quantities and have bounds. The initial parameters must stay within their limits if the recorded dataset has some values beyond the permitted bounds.

Two challenging cases are considered and tested:

Case 1) 6 erroneous parameters shown in Table. 
Table 1. Comparison results of parameter estimation in pu with and without adaptive weights, where the values shown in the brackets represent their standard deviations.

\begin{tabular}{|c|c|c|c|}
\hline $\begin{array}{c}\text { True } \\
\text { parameters }\end{array}$ & $\begin{array}{c}\text { Wrong } \\
\text { values }\end{array}$ & $\begin{array}{c}\text { Estimated } \\
\text { without adaptiveness }\end{array}$ & $\begin{array}{c}\text { Estimated } \\
\text { with adaptiveness }\end{array}$ \\
\hline$X_{d}=0.1218$ & 0.2 & $\mu=0.1938(\sigma=0.036)$ & $\mu=0.127(\sigma=0.004)$ \\
\hline$M=0.7568$ & 0.9 & $\mu=0.7628(\sigma=0.015)$ & $\mu=0.7617(\sigma=0.012)$ \\
\hline$T^{\prime} d=29.7$ & 32 & $\mu=32.5(\sigma=0.14)$ & $\mu=32.0(\sigma=0.16)$ \\
\hline$K F=0.063$ & 0.1 & $\mu=0.0622(\sigma=0.001)$ & $\mu=0.0623(\sigma=0.002)$ \\
\hline$K A=60$ & 40 & $\mu=70.20(\sigma=10.8)$ & $\mu=60.48(\sigma=0.32)$ \\
\hline$R D=1$ & 0.3 & $\mu=1(\sigma=0.001)$ & $\mu=1(\sigma=0.001)$ \\
\hline
\end{tabular}

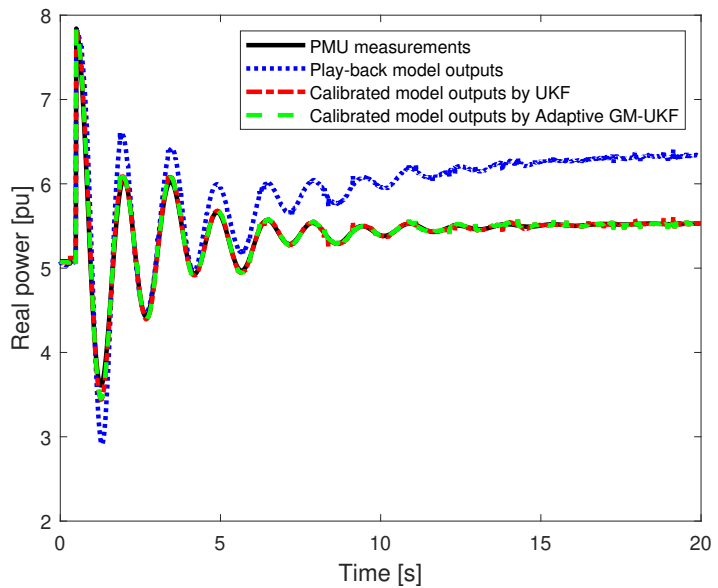

Figure 3. Real power response using calibrated parameters with non-Gaussian noise.

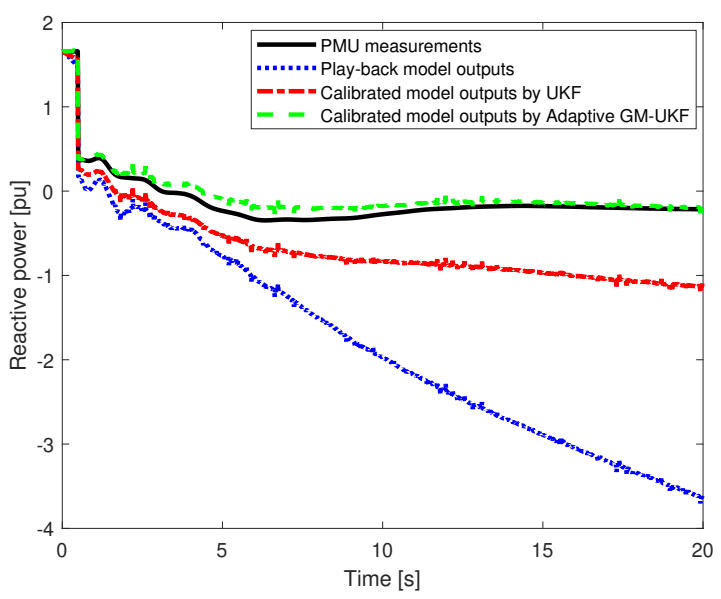

Figure 4. Reactive power response using calibrated parameters with non-Gaussian noise.

1, where $K A$ has strong correlations with $K F$ and $X_{d}$; the system process and measurement noises for all methods are simulated by random variables with zero mean and covariance matrix $10^{-6} \boldsymbol{I}$ of appropriate dimensions. Note that, the set up is different from the true measurement covariance matrix. This is because

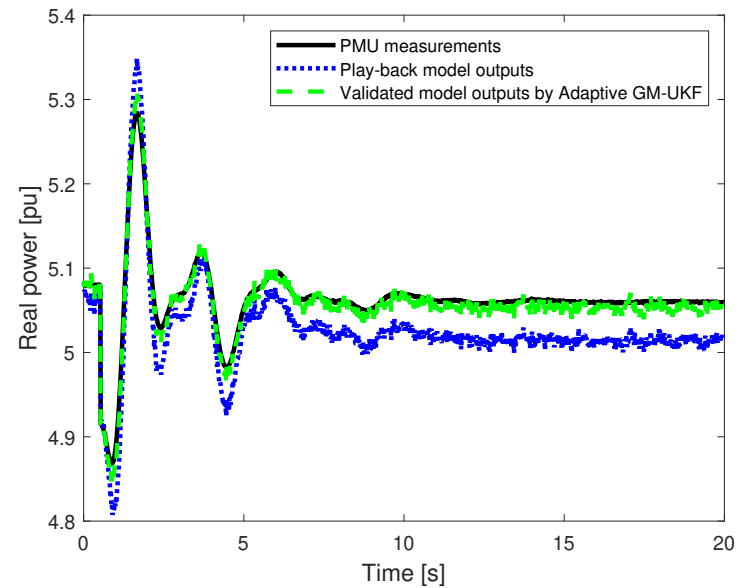

Figure 5. Validation of real power response using calibrated parameters when transmission line $15-16$ is switched off at $t=0.5 \mathrm{~s}$.

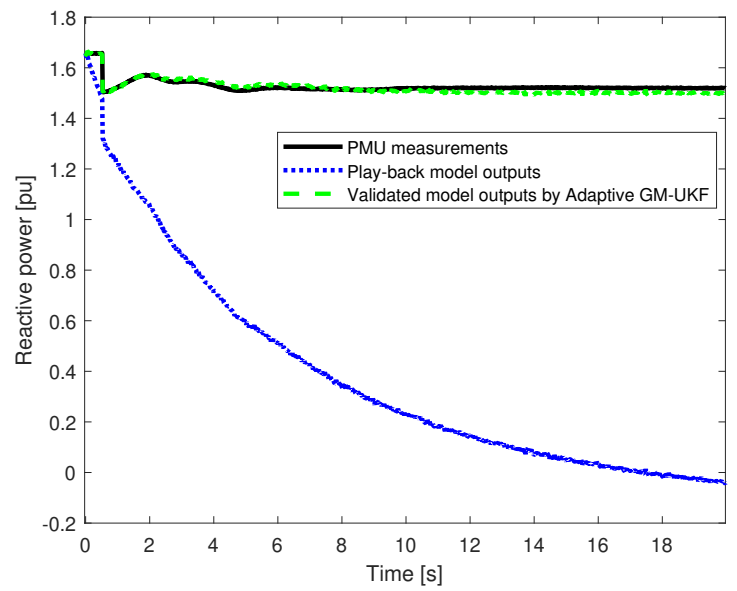

Figure 6. Validation of reactive power response using calibrated parameters when transmission line $15-16$ is switched off at $t=0.5 \mathrm{~s}$.

in practice, we do not know the true value of the measurement error and the developed methods should deal with the unknown noise.

Case 2) the occurrence of non-Gaussian measurement noise [21], where a bimodal Gaussian 


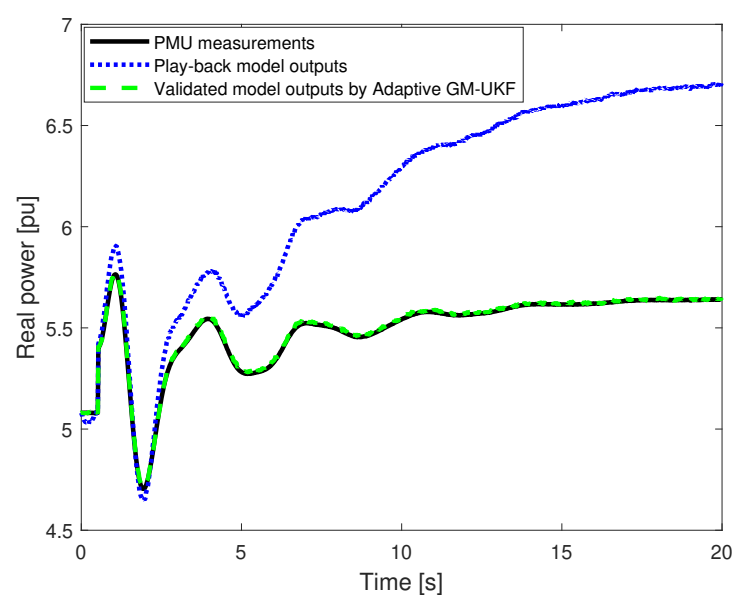

Figure 7. Validation of real power response using calibrated parameters when transmission line $29-38$ is switched off at $t=0.5$ s.

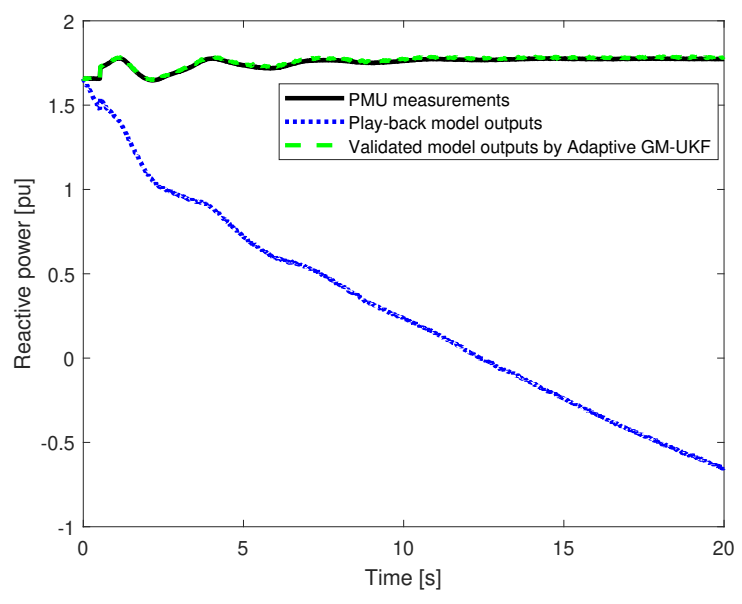

Figure 8. Validation of reactive power response using calibrated parameters when transmission line $29-38$ is switched off at $t=0.5$ s.

mixture model with zero means, covariance matrices of $10^{-6} \boldsymbol{I}$ and $10^{-4} \boldsymbol{I}$ and weights of 0.9 and 0.1 , are assumed for measurement noise with appropriate dimensions.

The results for Case 1 are displayed in Table. 1 and Figs. 1-2, where $\mu$ and $\sigma$ represent the mean and standard deviation of each parameter. In Table. 1 , only the results for GM-UKF with and without the adaptive weights derived from the sensitivity analysis are shown. It can be found that due to the strong dependency among parameters $K A, K F$ and $X_{d}$, the direct augmenting 6 parameters with system states for joint estimation will yield local optimal solutions of $K A$ and $X_{d}$. By contrast, with the derived adaptive weights and the strategy of breaking down the parameter dependence, the proposed adaptive GM-UKF can achieve much higher accuracy of calibrating the suspicious parameters. By further looking at the standard deviation for each estimated parameter, it is observed due to the lack of capability in dealing with correlated parameters, the method without adaptive weights has much larger variances. This is expected as the searching space for it is quite random and as a result, a good solution may be obtained sometimes. However, the solutions are not satisfactory most of the time. By contrast, with the adaptive weights, the correlations among erroneous parameters can be addressed and the search space is around the optimal values, yielding small standard deviations of the estimated parameters. The comparison results validate the effectiveness of the proposed adaptive weights in enhancing the parameter calibrations. The conclusion can be also validated by the results in Figs. 1-2, where the proposed method with adaptive weights gets much better matches for the real and reactive power than other alternatives.

For the Case 2, in the presence of non-Gaussian noise, EnKF has serious issues of getting converged results. Although UKF does not diverge, their results are strongly biased, see the comparison results between Figs. 1-2 and Figs. 3-4. The results are expected because UKF and EnKF are derived based on Gaussian assumption and the non-Gaussian noise significantly deteriorates their estimation efficiency. By contrast, thanks to the adpativeness and robustness of the proposed method, it can filter out the non-Gaussian noise and its performance is slightly affected.

To further validate the effectiveness of the calibrated parameters for different scenarios, two additional events have been simulated, including the transmission line switches at 15-16 and 29-38, respectively at $t=0.5 \mathrm{~s}$. It can be found from Figs. 5-8 that the real and reactive responses using the calibrated parameters would yield quite similar outcomes to the actual ones captured by PMU measurements. The tests using other events show a similar conclusion. As a result, the performance of the proposed method can be effectively validated.

\section{Conclusion}

This paper proposes a robust and adaptive nonlinear UKF for generator parameter calibration. The generalized nonlinear regression form is first derived that allows us to derive adaptive weights from the gPC-based ANOVA method. In addition, new strategy is developed to address the strong dependency between suspicious parameters. Finally, a generalized regression form that considers suspicious parameters and system states simultaneously is developed for robust filtering. 
Simulation results on the IEEE 39-bus system validate the effectiveness and robustness of the proposed approach under various conditions. Further work will be on testing the developed method using realistic power plant and field PMU measurements.

\section{References}

[1] NERC, "Standard MOD-026-1-Verification of models and data for generator excitation control system or plant volt/var control functions," 2014.

[2] NERC, "Standard MOD-027-1-Verification of models and data for turbine/governor and load control or active power/frequency control functions," 2013.

[3] Z. Huang, P. Du, D. Kosterev, and S. Yang, "Generator dynamic model validation and parameter calibration using phasor measurements at the point of connection,' IEEE Trans. Power Syst., vol. 28, no. 2, pp. 1939-1949, May 2013.

[4] IEEE Guide for Synchronous Generator Modeling Practices in Stability Analyses. Piscataway, NJ, USA: IEEE, 1991, pp. 1110-1991.

[5] Z. Huang, T. B. Nguyen, D. Kosterev, R. T. Guttromson, "Model validation of power system components using hybrid dynamic simulation," in Proc. IEEE PES Transm. Distrib. Conf. Expo., 2008, pp. 153-160.

[6] S. Benchluch and J. Chow, "A trajectory sensitivity method for the identification of nonlinear excitation system models," IEEE Trans. Energy Conv., vol. 8, no. 2, pp. 159-164, Jun. 1993.

[7] I. A. Hiskens, "Nonlinear dynamic model evaluation from disturbance measurements," IEEE Trans. Power Syst., vol. 16, no. 4, pp. 702-710, Nov. 2001.

[8] J. B. Zhao, et. al, "Power system dynamic state estimation: motivations, definitions, methodologies and future work," IEEE Trans. Power Syst., vol. 34, no. 4, pp. 3188-3198, Jul. 2019.

[9] B. Mogharbel, L. Fan, Z. Miao, "Least squares estimation-based synchronous generator parameter estimation using PMU data," IEEE Power and Energy Society General Meeting, pp. 1-5, 2015.

[10] L. Fan, Y. Wehbe, "Extended Kalman filtering based real-time dynamic state and parameter estimation using PMU data," Electric Power Systems Research, vol. 103, pp. 168-177, 2013.

[11] A. Mam, B. C. Pal, and A. K. Singh, "Estimating dynamic model parameters for adaptive protection and control in power system," IEEE Trans. Power Syst., vol. 30, no. 2, pp. 829-839, 2015.

[12] G. Valverde, E. Kyriakides, G. T Heydt, V. Terzija, "Nonlinear estimation of synchronous machine parameters using operating data," IEEE Trans. Energy Conversion, vol. 26, no. 3, pp. 831-839, 2011.

[13] R. Huang, et. al, "Calibrating Parameters of Power System Stability Models using Advanced Ensemble Kalman Filter," IEEE Trans. Power Syst., vol. 33, no. 3, pp. 2895-2905, 2018.

[14] C. Tsai, et al., "Practical considerations to calibrate generator model parameters using phasor measurements," IEEE Trans. Smart Grid, vol. 8, no. 5, pp. 2228-2238, Sep. 2017.
[15] Y. Xu, et al., "Response-surface-based bayesian inference for power system dynamic parameter estimation," IEEE Trans. Smart Grid, vol. 10, no. 6, pp. 5899-5909, 2019.

[16] P. Sauer, M. A. Pai. Power system dynamics and stability. Urbana, 1998.

[17] J. Foo, G.E. Karniadakis, "Multi-element probabilistic collocation method in high dimensions", J. Comput. Phys., vol. 229, no. 5, pp. 1536-1557, 2010.

[18] X. Yang, M. Choi, G. Lin, G. E. Karniadakis, "Adaptive ANOVA decomposition of incompressible and compressible flows", J. Comput. Phys., vol. 231, no. 4, pp. 1587-1614, 2012.

[19] J. B. Zhao, L. Mili, "A robust generalized-maximum likelihood unscented Kalman filter for power system dynamic state estimation," IEEE Journal of Selected Topics in Signal Processing, vol. 12, no. 4, pp. 578-592, 2018.

[20] IEEE PES TF on Benchmark System for Stability Controls, "Benchmark systems for small-signal stability analysis and control," Aug. 2015.

[21] S. Wang, J. B. Zhao, Z. Huang, R. Diao, "Assessing Gaussian assumption of PMU measurement error using field data," IEEE Trans. on Power Delivery, vol. 33, no. 6, pp. 3233-3236, 2018. 